\title{
Pulmonary, ocular and cutaneous Kaposi sarcoma in a HIV-infected patient
}

\author{
Mihaela Ionică , Șerban Benea, Alina Cozma, Lia Cavaropol, Elisabeta Benea \\ From The 10th Edition of the Scientific Days of the National Institute for Infectious Diseases "Prof Dr Matei \\ Bals" \\ Bucharest, Romania. 15-17 October 2014
}

\section{Background}

Pulmonary Kaposi sarcoma involvement is frequent in HIV-infected patients, and the diagnosis is more difficult in severe immunosuppressed. On the other hand, HIVinfected patients tend to develop malignant tumors, such as Kaposi sarcoma. Although cutaneous involvement is the most common manifestation, extracutaneous involvement is also possible.

\section{Case report}

We present the case of a 33 year-old male patient, HIV infected, with virological failure, who also had cutaneous (proved by histopathological exam of a cutaneous specimen) and ocular Kaposi sarcoma, prolonged fever and some pulmonary symptoms: cough, dyspnea, hypoxemia. Using bronchoscopy and pulmonary CT scan, we were able to diagnose the pulmonary involvement of Kaposi sarcoma.

\section{Conclusion}

Initial antibiotic and later antifungal therapy did not improve the pulmonary symptomatology (chest X-ray revealed bilateral bronchopneumonia); the evolution was slowly favorable only after the optimal control of HIV infection by antiretroviral therapy.

\section{Consent}

Written informed consent was obtained from the patient for publication of this Case report and any accompanying images. A copy of the written consent is available for review by the Editor of this journal.

* Correspondence: mihaela_ionica@yahoo.com

National Institute for Infectious Diseases "Prof. Dr. Matei Balş", Bucharest, Romania 